\title{
Origin of the highest energy cosmic rays
}

\author{
Peter L. Biermann ${ }^{\mathrm{a}}$, Eun-Joo Ahn ${ }^{\mathrm{b}}$, Gustavo Medina-Tanco ${ }^{\mathrm{c}}$, and Todor Stanev ${ }^{\mathrm{d}}$ \\ aMax Planck Institut für Radioastronomie, Bonn, Germany \\ ${ }^{\mathrm{b}}$ Department of Physics, Seoul National University, Seoul, Korea \\ ' Institute of Astronomy and Geophysics, University of Sao Paulo, Sao Paulo, Brasil \\ ${ }^{\mathrm{d}}$ Bartol Research Institute, University of Delaware, Newark, DE, USA
}

Introducing a simple Galactic wind model patterned after the solar wind we show that back-tracing the orbits of the highest energy cosmic events suggests that they may all come from the Virgo cluster, and so probably from the active radio galaxy M87. This confirms a long standing expectation. Those powerful radio galaxies that have their relativistic jets stuck in the interstellar medium of the host galaxy, such as $3 \mathrm{C} 147$, will then enable us to derive limits on the production of any new kind of particle, expected in some extensions of the standard model in particle physics. New data from HIRES will be crucial in testing the model proposed here.

\section{Introduction}

The origin of the highest energy particles observed in the universe continues to present a major challenge to physicists. These particles reach energies as high as $310^{20} \mathrm{eV}$. If protons, their flux is expected to drop sharply at $510^{19} \mathrm{eV}$ due to the interaction with the microwave background, commonly referred to as the GreisenZatsepin-Kuzmin- or GZK-cutoff after its discoverers. However, the number of particles known to be beyond $10^{20} \mathrm{eV}$ continues to increase, with now 14 published [26,23,24], and a further $10 \mathrm{ex}-$ pected from new observations with HIRES and a reanalysis of the Yakutsk data.

There are three basic difficulties:

First, we need to find a site that can produce particles at such energies [10,4]. There is only one class that has been argued to require protons at such energies in the source, namely radio galaxies with powerful jets and/or hot spots, such as M87 3] or Cyg A. M87 has been under suspicion to be the primary source for ultra high energy cosmic rays for a long time, e.g., 9, 4]. Gamma ray bursts are another possible class.

Secondly, there is the additional difficulty of getting these particles to us, that is, overcom- ing the losses in the bath of the cosmological microwave background. That implies that the source should not be very much further than 20 Mpc.

The third difficulty is the explanation of the nearly isotropic distribution of the arrival directions of these events.

There are several ways out of these difficulties. Here we present the consequences of introducing a magnetic Galactic wind in analogy to the solar wind. The magnetic field of the wind bends the particle orbits without adding substantial travel time.

\section{A model for a magnetic galactic wind}

It has long been expected that our Galaxy has a wind, e.g., 6] akin to the solar wind [16]. Recent modelling [5] shows that such winds can be quite fast, and ubiquituous.

It seems plausible that this wind is powered by the combined action of cosmic rays and magnetic fields and starts in the hot phase of the interstellar medium seen in X-rays by ROSAT [21].

Parker has shown 16] that in a spherical wind the azimuthal part of the magnetic field quickly becomes dominant with $B_{\phi} \sim \sin \theta / r$ in polar 
coordinates.

Cosmic ray driving is similar to radiation driving of winds in massive stars, and so [18] magnetic fields can lead to an increase of the momentum of the wind.

The data on the sign of the azimuthal component show that in the part of the sky most relevant for calculating orbits of energetic charged particles in our Galaxy, the field points to the direction of galactic longitude about 90 degrees [20,12]. That means immediately that positively charged particles traced backwards have their origin above us, at high positive galactic latitudes.

We adopt the simplest possible model. We assume that the magnetic field in the galactic wind has a dominant azimuthal component, and ignore all other components. We assume that this azimuthal component has the same sign everywhere [11]. Most measures of magnetic field underestimate its strength. Therefore we will consider for reference a model which has a field strength near the Sun of 7 microGauss; this is close to the best estimates for the total local field 22. The second parameter, the distance to which this wind extends, is more uncertain: Our Galaxy dominates its near environment well past our neighbor, M31, the Andromeda galaxy, and might well extend its sphere of influence to half way to M81. Therefore we will adopt as outer the halo wind radius half the distance to M81, 1.5 Mpc.

\section{Tracing the path backwards}

To follow the particle trajectories in the Galactic halo we trace protons backwards, e.g., 22,13, 19] from their arrival direction at Earth. We use the 14 published cosmic ray events above $10^{20}$ $\mathrm{eV}$, the list from Watson (included in [26]) and the new list from AGASA [24]. There is a big uncertainty with the energy estimate of the highest energy Yakutsk [1] event, which we therefore exclude from the present analysis, and hence we arrive at a final tally of 13 events used.

The interesting result of these model calculations is that the directions of all tracks point North. All events are consistent with arising originally from Virgo A. Since these particles are assumed to be accelerated out of cosmic gas, about
1/10 of all particles may be Helium nuclei with the same energy per particle. If the two highest energy events are in fact He nuclei, all 13 events point within 20 degrees of Virgo A.

If Virgo A is indeed the acceleration site of the highest energy cosmic ray events, they all require additional systematic bending at a ten to twenty degree level. Such bending could be easily accomodated within the plausible magnetic field strength within the supergalactic sheet from here to Virgo [25:17.

How critical are our assumptions for these results?

The assumption of the symmetry of the magnetic field above and below the Galactic disk is important.

The value of the magnetic field, here adopted as 7 microGauss, for the wind near the Sun, is a key parameter. If the magnetic field were considerably weaker, the focussing would be largely removed.

The scale of the Galactic wind here $1.5 \mathrm{Mpc}$, is not a critical parameter, since the calculations show that most of the bending happens within the first few $100 \mathrm{kpc}$.

\section{Discussion and implications}

This particular model can be tested in several ways; some of these tests are direct tests with data, and others take the form of a consistency check:

The most important test is obviously with more data, and the HIRES data are soon to be released and will allow straight tests to be made. For instance, the apparent focussing is fully maintained at $510^{19} \mathrm{eV}$.

The very concept of a magnetic wind, driven by cosmic rays, but with an initial magnetic field as strong as that in the disk, needs to be examined more closely.

There is one important consequence: If the model proposed here could be confirmed, then it would constitute strong evidence that all powerful radiogalaxies produce high energy cosmic rays, and that they do this at a good fraction of their total power output. This then implies that compact radio galaxies [14, 8] do provide a good test 
bed for particle interactions, since they have a large screen of interstellar gas around the radio hot spots and jets as seen in mm-wavelength radio data [15]. There the paradigm is materialized of having a gigantic accelerator, and a beam dump. These radio galaxies may be used for particle interaction experiments in the sky. If a significant correlation in arrival direction between ultra high energy cosmic rays and this specific class of radio quasars could be confirmed [8], then properties of new particles could be constrained.

In summary, we propose here that a very simple model for a Galactic wind rather analoguous to the Solar wind, may allow particle orbits at $10^{20}$ $\mathrm{eV}$ to be bent sufficiently to allow "super-GZK" particles to get here from M87, and also explain the apparent isotropy in arrival directions.

Acknowledgments. This work was started at the 1999 astrophysics summer school at the Vatican Observatory; both E.-J.A. and P.L.B. are grateful to George Coyne, S.J. and his colleagues for inviting us and for the fruitful and inspiring atmosphere at these schools. P.L.B, G.M.-T. and T.St. would like to acknowledge also useful discussions with Rainer Beck, Venya Berezinsky, Pasquale Blasi, E. Boldt, Glennys Farrar, J.L. Han, Anatoly Ivanov, Randy Jokipii, Frank Jones, Hyesung Kang, Phil Kronberg, Martin Lemoine, Friedrich Meyer, Hinrich Meyer, Biman Nath, Dongsu Ryu, Michael Salomon, Günther Sigl, Alan Watson, Arnold Wolfendale and many others. We thank Phil Kronberg for a careful reading of an earlier manuscript. Work with P.L.B. is partially supported by a DESY-grant, G.M.-T. is partially supported by the Brazilian agencies FAPESP and CNPq, T.St. is supported by NASA grant NAG5-7009, and PLB and TST have jointly a grant from NATO.

\section{REFERENCES}

1. Antonov, E.E. et al.: 1999 JETP Letters 69, 650.

2. Beck, R., Brandenburg, A., Moss, D., Shukurov, A. \& Sokoloff, D.: 1996 Ann. Rev. Astron. $\&$ Astrophys. 34, 155.

3. Biermann, P.L. \& Strittmatter, P.A.: 1987 Astrophys. J. , 322, 643.
4. Biermann, P.L.: 1997 J. Phys. G: Nucl. Part. Phys., 23, 1.

5. Breitschwerdt, D. \& Komossa, S.: 1999, in Astrophys. \& Sp. Sc., Proc. "Astrophys. Dynamics", Eds. D. Berry et al. (in press)

6. Burke, J.A.: 1968, Month. Not. Roy. Astr. Soc. 140, 241.

7. Cunningham, G. et al.: 1980, Astrophys. J. 236, L71

8. Farrar, G.R. \& Biermann, P.L.: 1998 Phys. Rev. Lett., 81, 3579.

9. Ginzburg, V.L. \& Syrovatskii, S.I.: 1964, The origin of cosmic rays, Pergamon Press, Oxford, Russian edition 1963

10. Hillas, A.M.: 1984 Ann. Rev. Astron. Astrophys., 22, 425.

11. Krause, F. \& Beck, R.: 1998 Astron. \& Astroph. 335, 789

12. Kronberg, P.P.: 1994 Rep. Prog. Phys., 57, 325.

13. Medina Tanco, G.A.: 1998c, Astrophys. J. Letters 505, L79.

14. O'Dea, C.P.: 1998 Publ. Astron. Soc. Pacific 110, 493 - 532

15. Papadopoulos, P.P. et al.: 1999 Astrophys. J. in press; astro-ph/9908286 Werf, S.

16. Parker, E.N.: 1958 Astrophys. J. 128, 664.

17. Ryu, D., Kang, H. \& P.L. Biermann: 1998 Astron. ES Astroph. 335, 19.

18. Seemann, H. \& Biermann, P.L.: 1997 Astron. E Astroph. 327, 273; astro-ph/9706117

19. Sigl, G., Biermann, P.L:: 1999 Astroparticle Physics 10, 141 - 156; astro-ph/9806283

20. Simard-Normandin, M. \& Kronberg, P.P.: 1979 Nature 279, 115

21. Snowden, S.L. et al.: 1997 Astrophys. J. 485, 125 .

22. Stanev, T.: 1997 Astrophys. J. 479, 290.

23. Takeda, M. et al. : 1998 Phys. Rev. Letters, 81, 1163.

24. Takeda, M. et al.: 1999 Astrophys. J. 522, 225 ; astro-ph/9902239.

25. Vallée, J.P.: 1990 Astron. J. 99, 459.

26. Wiebel-Sooth, B. \& Biermann, P.L:: 1999 Landolt-Börnstein, vol. VI/3c, Springer Verlag, p. 37. 